\title{
ON WILKER AND HUYGENS TYPE INEQUALITIES
}

\author{
EDWARD NEUMAN
}

Abstract. Trigonometric inequalities, which have been obtained by J. Wilker [11] and C. Huygens [4], have attracted attention of several researchers (see, e.g., [1], [3], [6], [7], [8], [9], [10], [12], [13], [14], [15], [16]). In this paper we offer several refinements and generalizations of these results.

Mathematics subject classification (2010): 26D05, 33B10.

Keywords and phrases: inequalities, trigonometric functions, hyperbolic functions, Wilker's inequality, Huygen's inequality..

\section{REFERENCES}

[1] A. BARICZ, J. SÁNDOR, Extensions of generalized Wilker inequality to Bessel functions, J. Math. Inequal. 2, 3 (2008), 397-406.

[2] P. S. Bullen, A Dictionary of Inequalities, Pitman Monographs and Surveys in Pure and Applied Mathematics, Vol. 97, Addison Wesley Longman Limited, Longman, Harlow, 1998.

[3] B. -N. GuO, B. -M. QIAO, F. QI, W. LI, On new proofs of Wilker inequalities involving trigonometric functions, Math. Inequal. Appl. 6, 1 (2003), 19-22.

[4] C. Huygens, Oeuvres Completes 1888-1940, Société Hollandaise des Science, Haga.

[5] D. S. Mitrinović, Analytic Inequalities, Springer-Verlag, Berlin, 1970.

[6] E. Neuman, One- and two-sided ineualities for Jacobian elliptic functions and related results, Integral Transform. Spec. Funct. 21, 6 (2010), 399-407.

[7] E. NEUMAN, J. SÁNDOR, On some inequalities involving trigonometric and hyperbolic functions with emphasis on the Cusa-Huygens, Wilker and Huygens inaequalities, Math. Inequal. Appl. 13, 4 (2010), 715-723.

[8] I. Pinelis, L'Hospital rules of monotonicity and Wilker-Anglesio inequality, Amer. Math. Monthly 111(2004), 905-909.

[9] J. SÁNDOR, M. BenCZE, On Huygen's trigonometric inequality, RGMIA Res. Rep. Collection, 8, 8 (2005), Article 14.

[10] J. S. Sumner, A. A. Jagers, M. Vowe, J. Anglesio, Inequalities involving trigonometric functions, Amer. Math. Monthly 98 (1991), 264-267.

[11] J. B. Wilker, Problem E 3306, Amer. Math. Monthly 96 (1989), 55.

[12] S. WU, A. BARICZ, Generlizations of Mitrinović, Adamović and Lazarevic's inequalities and their applications, Publ. Math. Debrecen 75 3-4 (2009), 447-458.

[13] S. - H. Wu, H. M. SRIVASTAVA, A weighted and exponential generalization of Wilker's inequality and its applications, Integral Transform. Spec. Funct. 18, 8 (2007), 525-535.

[14] L. ZHU, A new simple proof of Wilker's inequality, Math. Inequal. Appl., 8 (2005), No. 4, 749-750.

[15] L. ZHU, On Wilker-type inequalities, Math. Inequal. Appl., 10, 4 (2007), 727-731.

[16] L. Zhu, Some new Wilker type inequalities for circular and hyperbolic functions, Abstract Appl. Analysis, Vol. 2009, Article ID 485842, 9 pages. 